\title{
Estudo comparativo entre protocolos para profilaxia da trombose venosa profunda: uma nova proposta
}

\author{
Deep venous thrombosis prophylaxis protocols comparative study: a new proposal
}

\author{
Jorge Lorenzoni Moulim ${ }^{1}$ \\ Marconi Lima Sobreira ${ }^{2}$ \\ Rafael Demarchi Malgor ${ }^{3}$ \\ Cristiano Ricardo de \\ ABREU $^{1}$ \\ Emiliano SÁ Fortes de \\ Araújo ${ }^{1}$ \\ Aristides Augusto \\ Palhares Neto ${ }^{4}$
}

Trabalho realizado no Hospital das Clínicas da Faculdade de Medicina de Botucatu Universidade Estadual Paulista (UNESP), Botucatu, SP, Brasil.

Artigo submetido pelo SGP (Sistema de Gestão de Publicações) da RBCP.

Artigo recebido: $27 / 6 / 2010$ Artigo aceito: 26/7/2010

\begin{abstract}
RESUMO
Introdução: O artigo compara os protocolos Davison-Caprini (americano) e Sandri (brasileiro) para profilaxia da trombose venosa profunda (TVP), buscando a elaboração de um novo protocolo, mais abrangente e aplicável. Método: Foi realizado um estudo prospectivo, durante um ano, abrangendo 212 pacientes, comparando os protocolos quanto à estratificação de risco de TVP, e ao tipo de profilaxia indicado. Um novo protocolo foi proposto, aplicado e comparado aos anteriores. Resultados: Obteve-se um novo protocolo, denominado Sandri modificado, que restringe a heparina a um grupo seleto de pacientes, semelhante ao americano, mantendo-se adequado tanto para cirurgias estéticas quanto reparadoras. Conclusões: A baixa adesão, por parte dos cirurgiões plásticos, ao uso rotineiro da profilaxia de TVP, espelha a escassez de informação sobre o tema. O protocolo Sandri modificado busca melhorar a compreensão e a aplicabilidade da profilaxia da TVP na cirurgia plástica.
\end{abstract}

Descritores: Trombose venosa/prevenção \& controle. Embolia e trombose/prevenção \& controle. Cirurgia plástica.

\section{SUMMARY}

Background: Compare Davison-Caprini (American) and Sandri (Brazilian) protocols for deep vein thrombosis (DVT) prophylaxis, suggesting a new protocol, more comprehensive and applicable. Method: A prospective study was conducted over a year, including 212 patients, comparing the protocols on DVT stratification risk, and the type of prophylaxis indicated. A new protocol was proposed, applied and compared to the previous. Results: The new protocol, modified Sandri, restricts the heparin to a select group of patients, similar to the American protocol, and remains suitable for both aesthetic and reconstructive surgery. Conclusions: The low adhesion of plastic surgeons to the routine use of DVT prophylaxis reflects lack of information concerning this topic. The Sandri modified protocol seeks improvement on the understanding and applicability of DVT prophylaxis in plastic surgery.

Descriptors: Venous thrombosis/prevention \& control. Embolism and thrombosis/prevention \& control. Plastic surgery.

1. Membro da Sociedade Brasileira de Cirurgia Plástica (SBCP); Cirurgião Plástico do Hospital das Clínicas da Faculdade de Medicina de Botucatu UNESP..

2. Doutor; Professor Doutor da Disciplina de Cirurgia Vascular da Faculdade de Medicina de Botucatu - UNESP.

3. Fellow da Mayo Clinic, Minnesota; Cirurgião Vascular.

4. Doutorado; Professor Doutor da Disciplina de Cirurgia Plástica da Faculdade de Medicina de Botucatu - UNESP, e membro da SBCP. 


\section{INTRODUÇÃO}

Arealidade atual nos traz uma busca constante pela perfeição técnica e maior segurança nos procedimentos. A trombose venosa profunda (TVP) e a embolia pulmonar (EP) permanecem como inimigos oportunistas que, pela baixa incidência, por vezes levam o cirurgião plástico a menosprezar os riscos.

Não existe uma incidência de TVP em cirurgia plástica que represente de forma fidedigna todo o conjunto de cirurgias, mas sim, estimativas de risco, específicas para cada tipo de procedimento. Segundo dados da literatura, a incidência de TVP no lifting facial é $0,35 \%^{1}$, na lipoaspiração é $0,6 \%^{2}$, em abdominoplastias é cerca de $1,3 \%{ }^{3,4}$ (abdominoplastia circunferencial e a associada a cirurgia intra-abdominal apresentam incidência de TVP, respectivamente, de 3,4 e $2,17 \%)^{5}$ e no retalho transverso do músculo reto abdominal (TRAM) monopediculado é $1,3 \%{ }^{6}$. Em relação à $\mathrm{EP}$, a incidência também não é definida: no lifting facial é estimada em $0,14 \%{ }^{1}$, em abdominoplastias, $0,8 \%^{3}$ (se associado a outras cirurgias, varia de $1,1 \%^{7}$ a $6,6^{8} \%^{8}$ ), na lipoaspiração, $1,1 \%^{2}$, e no retalho TRAM varia de 0,7 a $18,8 \% \%^{9,10}$.

Estudos atuais americanos concluíram que apenas a minoria dos cirurgiões plásticos usa a profilaxia de TVP de forma rotineira ${ }^{1,11}$. Buscando um protocolo de profilaxia que incluísse os pacientes de cirurgia plástica, em 2001, o American College of Chest Physicians (ACCP), no Consenso da Sexta Conferência em Terapia Antitrombótica, publicou uma ampla discussão sobre profilaxia de TVP e estratificou quatro categorias de risco, baseadas em idade, quadro clínico e fatores de risco ${ }^{11}$. Com base nesta publicação, e nos estudos de Caprini et al. ${ }^{12}$, Davison et al. ${ }^{13}$ desenvolveram um algoritmo para profilaxia de TVP em cirurgia plástica baseado em fatores de risco. Este protocolo, denominado DavisonCaprini, é considerado como grau 2C de recomendação pelo $\mathrm{ACCP}^{14}$. Trata-se do maior grau de recomendação que pode ser atribuído a dados não procedentes de estudos controlados e randomizados. Recentemente, foi demonstrada sua eficiência na estratificação de risco de TVP, onde $89,5 \%$ dos eventos tromboembólicos ocorreram em pacientes arrolados no grupo de risco muito alto ${ }^{15}$.

No Brasil, tem-se o protocolo de profilaxia publicado, em 2005 , por Sandri ${ }^{16}$, que também exibe condutas baseadas em estratificação de risco.

No presente estudo, o objetivo foi comparar os protocolos brasileiro e americano para profilaxia da TVP, ressaltando suas semelhanças e disparidades, buscando um novo protocolo, mais abrangente e aplicável.

\section{MÉTODO}

Foi realizado um estudo prospectivo, no período de março de 2007 a março de 2008, onde foram inclú́dos, consecutivamente, os pacientes submetidos à cirurgia plástica, estética e reparadora, no centro cirúrgico do Hospital das Clínicas da Faculdade de Medicina de Botucatu, Universidade Estadual Paulista (UNESP).

Todos os pacientes foram devidamente orientados e assinaram um termo livre e esclarecido, no qual consentiam com o protocolo de pesquisa, cujo formulário para coleta de dados está demonstrado no Anexo 1.

Tomou-se como critério de exclusão a recusa em participar do protocolo de pesquisa, bem como a discordância com o termo de consentimento.

A coleta dos dados foi realizada na véspera da cirurgia, tendo sido iniciada após aprovação pelo Comitê de Ética em Pesquisa (282/2007).

De acordo com o protocolo Sandri, os pacientes são classificados como risco baixo (caso a soma dos fatores de risco seja 0 ou 1 ), moderado (2, 3 ou 4), e alto (maior que 4 ), estando a profilaxia medicamentosa indicada nos riscos moderado e alto ${ }^{16}$.

Segundo as recomendações do protocolo americano de Davison-Caprini, os pacientes são classificados como risco baixo (caso a soma dos fatores de risco for 0 ou 1), moderado (2), alto (3 e 4) e muito alto (maior que 4), estando indicada a profilaxia medicamentosa apenas no risco muito alto ${ }^{13}$.

Como o protocolo Davison-Caprini apresenta um grupo a mais na estratificação de risco (o risco muito alto), separamos os dois protocolos em grupos que permitissem comparação, ou seja, de acordo com a indicação ou não da profilaxia medicamentosa. Sendo assim, cada protocolo foi dividido em dois grupos (Tabela 1):

- Sandri: risco baixo vs. riscos moderado e alto;

- Davison-Caprini: risco baixo, moderado e alto vs. risco muito alto.

Os pacientes foram avaliados quanto à estratificação de risco de TVP, pelos protocolos brasileiro e americano, observando-se as diferenças quanto à sua faixa de risco, e, consequentemente, quanto ao tipo de profilaxia indicado.

Com base nos resultados desta análise, foi proposto um novo protocolo (Sandri modificado), pelo qual os pacientes foram reavaliados, segundo suas categorias de indicação de tromboprofilaxia, e comparados aos resultados dos outros dois protocolos (Davison-Caprini e Sandri), expondo suas diferenças e possíveis vantagens.

Para análise estatística foram usados os testes estatísticos de quiquadrado e Fisher, considerando como nível de significância $p<0,05$.

\section{RESULTADOS}

No total foram avaliados 212 pacientes, sendo $28,3 \%$ do sexo masculino e $71,7 \%$ do sexo feminino, e idade média de 33,9 anos, com a mesma variando entre 1 dia (correção de mielomeningocele) e 101 anos (ressecção de carcinoma espinocelular de pavilhão auricular).

Quanto ao tipo de cirurgia realizada, $107(50,47 \%)$ foram de cunho estético e 105 (49,53\%), reparador.

De acordo com o protocolo Sandri, 29,72\% dos pacientes foram classificados no grupo de baixo risco, $61,32 \%$, no moderado, e 8,96\%, no alto risco. Quando esses pacientes foram avaliados segundo o protocolo Davison-Caprini, $0,47 \%$ foram considerados de baixo risco, $42,45 \%$ de risco moderado, $47,17 \%$ de alto risco e $9,91 \%$ apresentaram risco muito alto para complicações tromboembólicas (Figura 1).

Comparando os dois protocolos sob o ponto de vista da indicação ou não da profilaxia medicamentosa, observou-se 


\title{
Anexo 1 - Formulário para coleta de dados
}

\author{
FORMULÁRIO PARA PROFILAXIA TVP/TEP
}

\section{Fatores de risco:}

\section{Sandri}

O Idade $>60$ anos: 2

O Idade entre 40 e 60 anos: 1

O Obesidade ( $\left.\mid \mathrm{IMC}^{1}>30\right): 1$

O Insuficiência venosa ou edema de membros: 2

O TVP ou EP prévia: 2

$\mathrm{O}$ Uso de $\mathrm{ACHO} / \mathrm{TRH}^{2}: 1$

O Fumante: 1

O Queimadura: 2

O Viagens longas 10 dias antes: 1

O Imobilização prévia à cirurgia $>24 \mathrm{~h}: 2$

O Cirurgia $>1 \mathrm{~h}: 1$

O Posição Fowler: 1

O Dermolipectomia abdominal ou coxa: 1

O Prótese em glúteo coxa ou perna: 1

O Lipoaspiração: 1

O Cirurgias estéticas associadas: 1

O Reconstrução de mama com retalho: 1

O Outras cirurgias associadas: 2

BAIXO RISCO: 0 ou 1 ponto

MODERADO RISCO: 2 a 4 pontos

ALTO RISCO: $>4$ pontos

Total de Pontos:

O Baixo O Moderado O Alto

\section{Davison-Caprini}

O Anestesia geral ou cirurgia $>1 \mathrm{~h}: 2$

O Imobilização panturrilha (gesso): 2

O Restrição leito $>72 \mathrm{~h}: 2$

O Acesso venoso central: 2

O IAM ${ }^{3}$ prévio, ICC ${ }^{4}$, Sepse: 3 (cada)

O Retalho livre: 3

O Fx MMII ${ }^{5}$, trauma múltiplo: 5 (cada)

O Lesão medular: 5

O Idade $>60$ anos: 2

O Idade entre 40 e 60 anos: 1

O História TVP/EP: 3

O Gravidez ou $<1$ mês pós parto: 1

O Malignidade: 2

O Obesidade: 1

O Uso de ACHO/ TRH: 1

O Desordem de hipercoagulação: 3

BAIXO RISCO: 0 ou 1 ponto

MODERADO RISCO: 2 pontos

ALTO RISCO: 3 ou 4 pontos

MUITO ALTO RISCO: $>4$ pontos

Total de pontos:

O Baixo O Moderado O Alto O Muito alto

Profilaxia realizada? O SIM O NÃO

Cirurgia realizada:

Tempo de cirurgia: horas

Tipo de anestesia: O Geral O Raqui/ Peridural O Local + sedação

Complicações associadas à profilaxia? O SIM O NÂO

Observações:

1 Índice de massa corpórea

${ }^{2}$ Anticoncepcional hormonal oral/ Terapia de reposição hormonal

${ }^{3}$ Infarto agudo do miocárdio

${ }^{4}$ Insuficiência cardíaca congestiva

${ }^{5}$ Fratura em membros inferiores 
Tabela 1 - Subdivisão dos protocolos Sandri e Davison-Caprini em dois grupos, conforme a indicação ou não da profilaxia medicamentosa.

\begin{tabular}{ccc}
\hline & Profilaxia sem indicação & Profilaxia indicada \\
\hline Sandri & Risco baixo & Risco moderado e alto \\
Davison-Caprini & Risco baixo, moderado e alto & Risco muito alto \\
\hline
\end{tabular}

que, de acordo com o protocolo brasileiro (Sandri), 70,28\% dos pacientes teriam indicação de heparina profilática (riscos moderado e alto), enquanto que, seguindo o protocolo americano (Davison-Caprini), apenas 9,91\% a teriam (risco muito alto). Essa diferença foi significativa $(\mathrm{p}<0,01)$.

Analisando cirurgias estéticas e reparadoras separadamente, verificamos que, de acordo com o protocolo brasileiro, $79,4 \%$ das cirurgias estéticas e $60,9 \%$ das reparadoras receberiam a profilaxia medicamentosa, e que, de acordo com o americano, a receberiam apenas $1,86 \%$ das cirurgias estéticas e $18,09 \%$ das reparadoras. Essa diferença também foi estatisticamente significativa, com $\mathrm{p}<0,01$ (Figura 2).

Foi realizada, então, uma avaliação do protocolo brasileiro, considerando a indicação de profilaxia medicamentosa apenas para o grupo de alto risco (em detrimento do grupo de risco moderado). Observou-se que, neste caso, a heparinoterapia profilática seria administrada em $8,96 \%$ dos pacientes. Esta porcentagem não presenta diferença estatística $(p=0,86)$ se comparada ao protocolo americano, o qual indica a medicação para 9,91\% dos pacientes (Figura 3).

Mantendo, no protocolo Sandri, a indicação de profilaxia tromboembólica apenas para os pacientes de alto risco, comparou-se os protocolos Sandri e Davison-Caprini considerando separadamente as cirurgias estéticas e reparadoras, e foi visto que o protocolo americano indicaria a profilaxia medicamentosa em apenas 1,86\% dos pacientes de cirurgia estética, enquanto o brasileiro em 10,28\% $(\mathrm{p}<0,01)$. Por outro lado, o protocolo brasileiro indicaria a tromboprofilaxia medicamentosa em apenas 7,61\% das cirurgias reparadoras, enquanto o americano em 18,09\% $(\mathrm{p}<0,05)$ (Figura 3).

Acrescentaram-se, então, ao protocolo brasileiro, os três fatores de risco exclusivos do protocolo americano, de maior ocorrência nos pacientes: anestesia geral (86,3\% dos casos), malignidade $(6,6 \%)$ e restrição ao leito maior que 72 horas $(5,18 \%)$ (Tabela 2).

Todos os 212 pacientes foram avaliados por este protocolo modificado, denominado protocolo Sandri modificado ou Sandri M, e observou-se que, a profilaxia medicamentosa estaria indicada para $14,15 \%$ dos pacientes, valor que não apresenta diferença estatística quando comparado aos protocolos brasileiro $(8,96 \%)(p=2,3)$, e americano $(9,91 \%)(p=0,23)$ (Figura 4$)$.

Contudo, considerando cirurgias estéticas apenas, o novo protocolo indica a heparina para $10,28 \%$ dos pacientes, de forma que não há diferença estatística em relação ao brasileiro

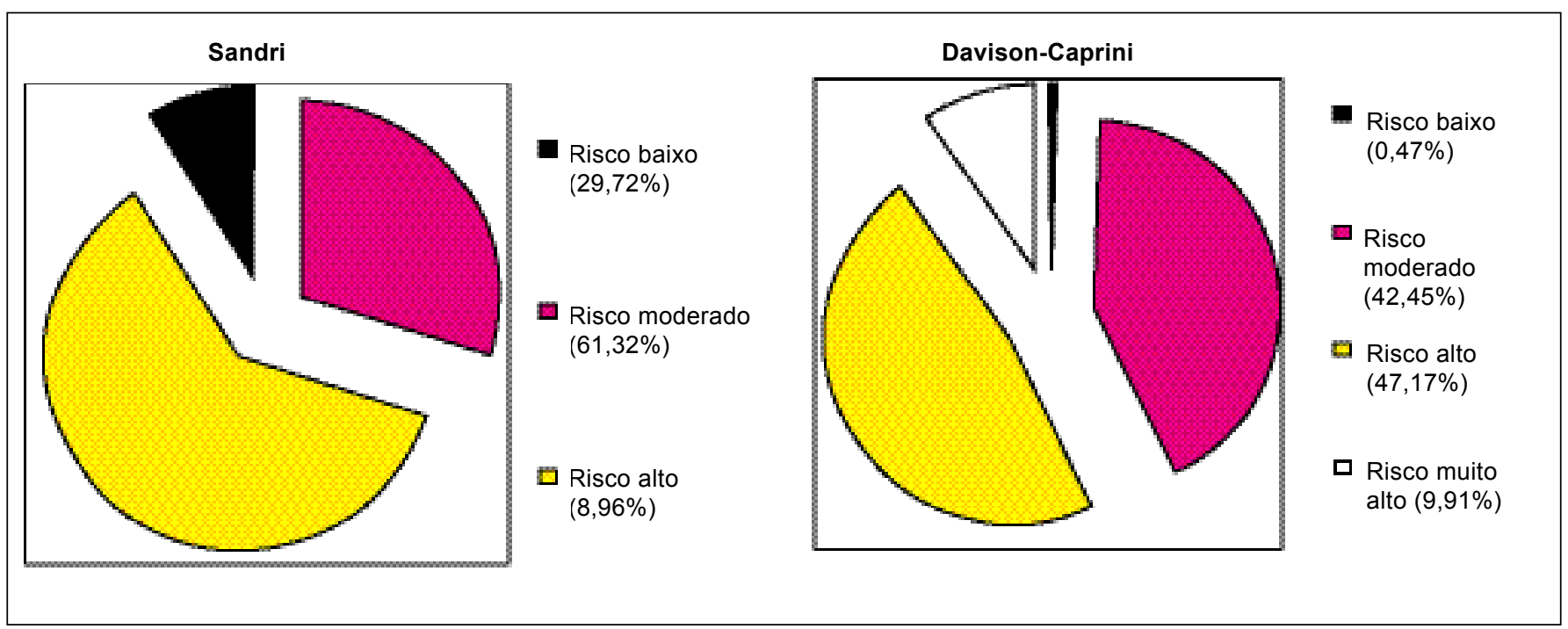

Figura 1 - Distribuição dos pacientes, segundo o grupo de risco, pelos protocolos Sandri e Davison-Caprini. 
$(10,28 \%)(p=1,0)$, mas sim em relação ao americano $(1,86 \%)$ $(\mathrm{p}<0,05)$ (Figura 4).

Além disso, abordando apenas as cirurgias reparadoras, o novo protocolo indica a tromboprofilaxia medicamentosa para $18,09 \%$ dos pacientes, havendo diferença estatística em relação ao Sandri $(7,61 \%)(p<0,05)$, e ausência da mesma em relação ao americano $(18,09 \%)(p=1,0)$ (Figura 4).

\section{DISCUSSÃO}

A busca constante pela melhoria de resultados e eliminação de riscos torna necessária a preocupação com as complicações tromboembólicas, que podem aumentar consideravelmente a morbidade e mortalidade no pós-operatório.

$\mathrm{O}$ uso da profilaxia incontestavelmente diminui a incidência de TVP $^{17}$. A profilaxia mecânica, caracterizada por compressão intermitente de panturrilhas, aliada ao uso de meias elásticas e à deambulação precoce, a reduz em até $60 \%$, devendo ser iniciada antes da indução anestésica ${ }^{17}$.

Por outro lado, a profilaxia química, caracterizada pela heparinoterapia profilática, diminui a incidência de TVP nos segmentos venosos proximais em $78 \%{ }^{11}$.

Apesar de todos esses benefícios da tromboprofilaxia, o ACCP constatou, em seu Consenso da Sexta Conferência em Terapia Anti-Trombótica, que muitos cirurgiões plásticos não usam tromboprofilaxia por acreditarem que a incidência

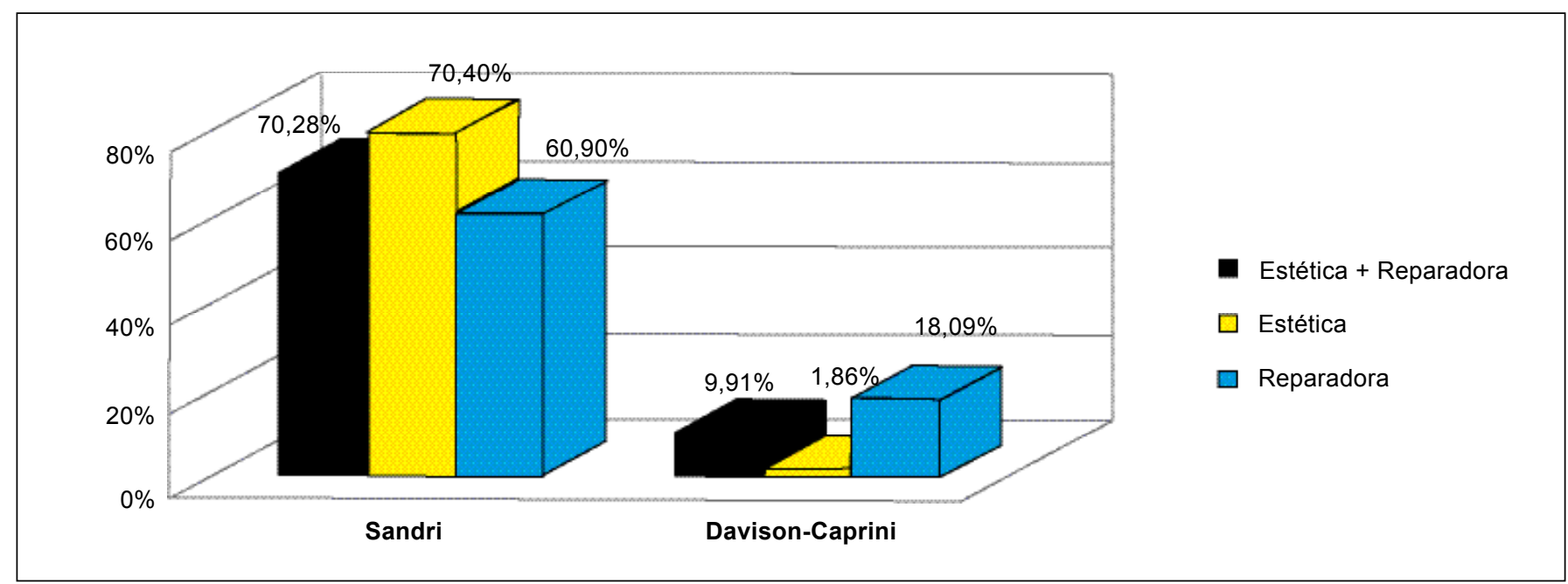

Figura 2 - Porcentagem de cirurgias com indicação de profilaxia medicamentosa, segundo os protocolos Sandri e Davison-Caprini.

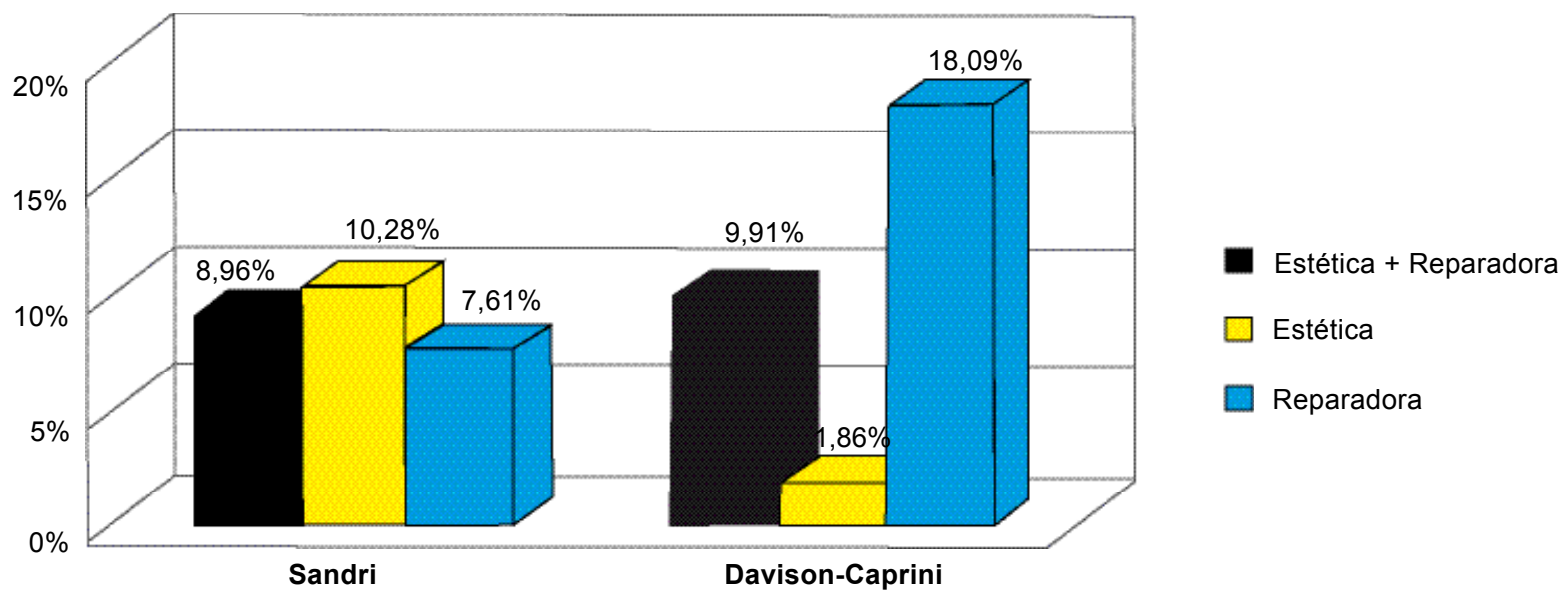

Figura 3 - Porcentagem de cirurgias com indicação de profilaxia medicamentosa, segundo os protocolos Sandri e Davison-Caprini, considerando, no protocolo Sandri, indicação de heparina apenas para o grupo alto risco. 
Tabela 2 - Fatores de risco do protocolo Davison-Caprini ausentes no protocolo Sandri, com suas respectivas ocorrências na amostra estudada.

\begin{tabular}{l|c|c}
\hline Fatores de Risco & Ocorrência absoluta & Ocorrência percentual \\
\hline Anestesia geral & 183 & $86,3 \%$ \\
\hline Malignidade & 14 & $6,60 \%$ \\
\hline Restrição ao leito $>$ 72h & 11 & $5,18 \%$ \\
\hline Imobilização panturrilha & 2 & $0,9 \%$ \\
\hline Lesão medular & 2 & $0,9 \%$ \\
\hline Acesso venoso central & 1 & $0,47 \%$ \\
\hline Fx MMII ${ }^{1}$ trauma múltiplo & 1 & $0,47 \%$ \\
\hline IAM prévio ${ }^{2}$, ICC ${ }^{3}$, sepse & 0 & $0 \%$ \\
\hline Retalho livre & 0 & $0 \%$ \\
\hline Gravidez ou $<1$ mês pós-parto & 0 & $0 \%$ \\
\hline Desordem de hipercoagulação & 0 & $0 \%$ \\
\hline
\end{tabular}

1 - FX MMII: Fratura de membros inferiores

2 - IAM prévio: Infarto agudo do miocárdio prévio

3 - ICC: Insuficiência cardíaca congestiva

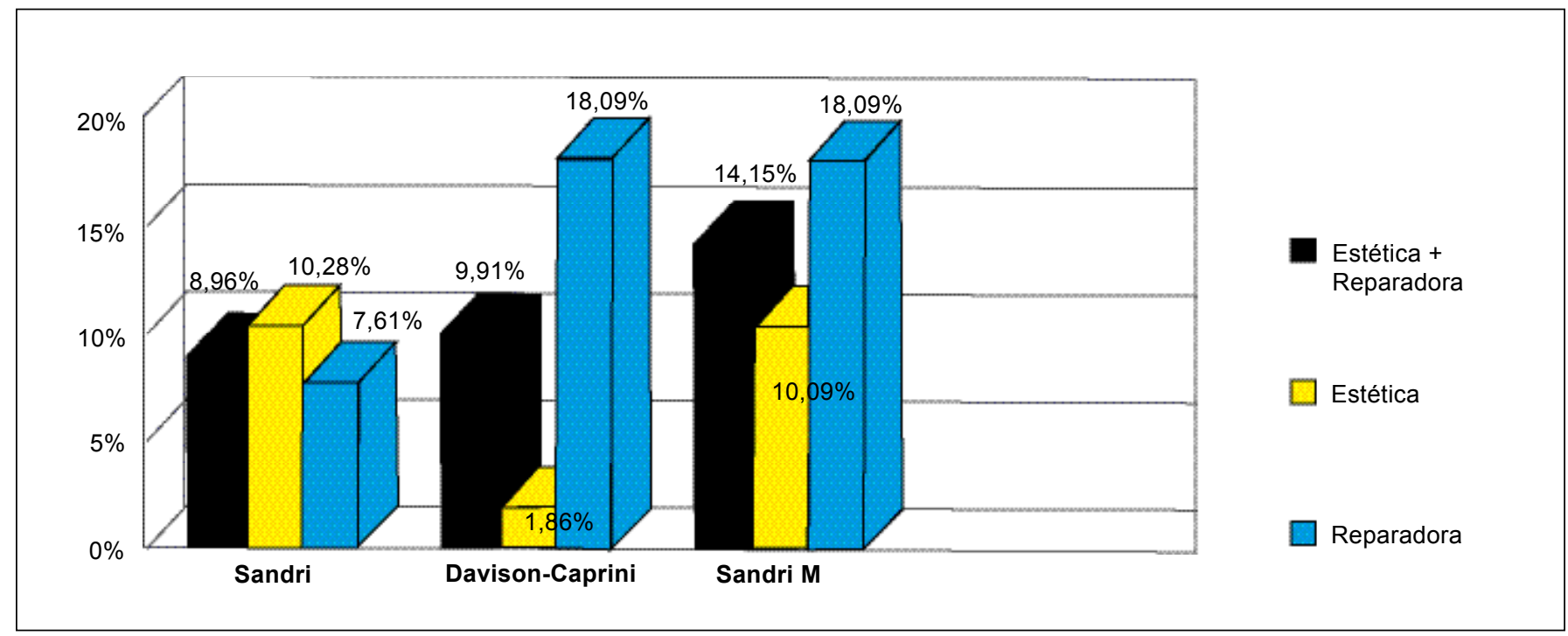

Figura 4 - Porcentagem de cirurgias com indicação de profilaxia medicamentosa, segundo os protocolos da Sandri, Davison-Caprini e Sandri M.

de TVP entre pacientes hospitalizados e naqueles em pósoperatório é tão baixa, que dispensa o uso da profilaxia. Outra possível razão seria a preocupação com sangramentos ${ }^{11}$.

A respeito do receio de hemorragias, estudos sugerem não haver aumento significativo do risco de sangramento com uso de heparina de baixo peso molecular (HBPM) ou nãofracionada ${ }^{11,18-21}$, contudo, há um risco ligeiramente maior de pequenos sangramentos com o uso da heparina não-fracionada, se comparada com a de baixo peso molecular ${ }^{11,18}$. O uso da HBPM como profilaxia seria, inclusive, não recomendado, devido ao maior risco de sangramento, necessidade eventual de monitorização do TTPA (tempo de tromboplastina parcial ativada) e potencial maior de indução de trombocitopenia ${ }^{16,22}$. A desvantagem da HBPM seria o seu maior custo ${ }^{22}$.

De acordo com o protocolo Sandri, estaria indicada a profilaxia medicamentosa nos riscos moderado (HBPM na 


$\quad$ PROTOCOLO Sandri M
$\quad$ Fatores de risco:
Idade >60 anos: 2
Idade entre 40 e 60 anos: 1
Obesidade ( IMC'>30): 1
Insuficiência venosa ou edema de membros: 2
TVP ou EP prévia: 2
Uso de ACHO/ TRH² : 1
Fumante: 1
Queimadura: 2
Viagens longas 10 dias antes: 1
Imobilização prévia à cirurgia>24h: 2
Posição Fowler: 1
Dermolipectomia abdominal ou coxa: 1
Prótese em glúteo coxa ou perna: 1
Lipoaspiração: 1
Cirurgias estéticas associadas: 1
Reconstrução de mama com retalho: 1
Outras cirurgias associadas: 2
Cirurgia >1h ou anestesia geral: 1
Restrição ao leito maior que $72 \mathrm{~h}: 2$
Malignidade: 2
BAIXO RISCO: 1 ponto
MODERADO RISCO: 2 a 4 pontos
ALTO RISCO: > 4 pontos
2 Antice de massa corpórea
MEDIDAS DE PROFILAXIA:
-Não farmacológicas: se aplicam a TODOS os pacientes:
cotidiana.
anestésica)
. Mobilização precoce
. Meias elásticas (Iniciar antes da indução anestésica e manter
até a deambulação cotidiana)
- Farmacológicas: nos pacientes de risco alto:
HBPM - Enoxaparina (Clexane) 40 mg subcutâneo, com início
-

Figura 5 - Protocolo Sandri Modificado para profilaxia de TVP.

indução se anestesia geral, ou $2 \mathrm{~h}$ após a anestesia no caso de punção lombar, mantendo dose diária até a deambulação) e alto (HBPM preferencialmente $12 \mathrm{~h}$ antes da cirurgia, mantendo dose diária até a deambulação) ${ }^{16}$. Por outro lado, o protocolo Davison-Caprini indicaria a profilaxia medicamentosa com heparina apenas no risco muito alto (enoxaparina $40 \mathrm{mg}$ subcutânea, com a $1^{\mathrm{a}}$ dose após $12 \mathrm{~h}$ de pós-operatório, e dose diária de manutenção até a deambulação cotidiana) ${ }^{13}$. Em ambos protocolos, a profilaxia mecânica está sempre indicada, e consiste de compressão pneumática intermitente, mobilização precoce e uso de meias elásticas.

No presente estudo comparativo, constatou-se a significante diferença (Sandri: 70,28\%; Davison-Caprini: 9,91\%) que os protocolos apresentam quanto à indicação da profilaxia medicamentosa (Figura 2). Esse resultado é consequência da heterogeneidade na categorização de risco dos pacientes entre os dois protocolos estudados, demonstrando a divergência de opiniões a respeito da indicação da profilaxia tromboembólica, particularmente a medicamentosa.

Quando se modifica o protocolo Sandri, indicando a profilaxia medicamentosa apenas para o grupo de alto risco, obtêm-se uma percentagem de indicação da heparina sem diferença estatística em comparação ao protocolo americano (Sandri: 8,96\%; Davison-Caprini: 9,91\%; $\mathrm{p}=0,86$ ). Desta forma, os protocolos seriam equivalentes quanto à indicação da tromboprofilaxia (Figura 3).

Analisando os protocolos Sandri (já modificado para profilaxia medicamentosa indicada apenas no grupo de alto risco) e Davison-Caprini, nota-se que o primeiro indica a profilaxia medicamentosa preferencialmente em cirurgias estéticas em detrimento das reparadoras, enquanto o americano a indica mais em cirurgias reparadoras, e pouco em estéticas. Isso ocorre porque o protocolo brasileiro leva em consideração fatores de risco específicos para cirurgia estética, tais como: posição de Fowler, dermolipectomia abdominal ou crural, implante glúteo, de coxa ou panturrilha, lipoaspiração e cirurgias estéticas associadas ${ }^{16}$. O protocolo americano, em contrapartida, pontua mais as situações clínicas desfavoráveis, presentes com maior frequência nas cirurgias reparadoras, tais como: acesso venoso central, retalho livre, malignidade, sepse e desordem de hipercoagulação ${ }^{13}$ (Anexo 1).

Buscou-se, então, um protocolo que atendesse de forma mais abrangente, tanto os pacientes submetidos a cirurgias estéticas quanto reparadoras. Optou-se por acrescentar ao protocolo brasileiro os 3 fatores de risco do protocolo americano com maior prevalência nos pacientes estudados: anestesia geral, malignidade e restrição ao leito maior que 72 horas (Tabela 2).

Comparando os resultados deste protocolo modificado (Sandri modificado ou Sandri M) com os protocolos de Sandri e Davison-Caprini, percebe-se sua equivalência com Davison-Caprini na indicação geral da profilaxia medicamentosa (Sandri M: 8,96\%; Davison-Caprini: 9,91\%; $\mathrm{p}=0,86$ ), e na indicação da profilaxia medicamentosa em cirurgias reparadoras (Sandri M: 18,09\%; Davison-Caprini:18,09\%; $\mathrm{p}=1,0$ ), bem como, nota-se sua equivalência com o protocolo de Sandri na indicação da profilaxia medicamentosa em cirurgias estéticas (Sandri M: 10,28\%; Sandri: 10,28\%; p=1,0). Dessa forma, essa nova versão do protocolo brasileiro seria equivalente ao americano na indicação geral da profilaxia medicamentosa, eliminando a carência desta indicação em cirurgias reparadoras (presente no Sandri), e em cirurgias estéticas (presente no Davison-Caprini) (Figura 4).

Neste novo protocolo, a HBPM seria aplicada, nos casos indicados, apenas $12 \mathrm{~h}$ após o procedimento, tal qual o americano. Isto porque, quando administrada no pré-operatório, aumenta levemente o risco de sangramento, se comparada à administração pós-operatória ${ }^{23}$.

Em suma, o novo protocolo proposto constitui-se do protocolo de Sandri acrescido das seguintes alterações (Figura 5):

- Indicação da profilaxia medicamentosa apenas para o grupo de alto risco, limitando o uso da heparina a um 
grupo mais seleto de pacientes, semelhante ao protocolo Davison-Caprini.

- Adição de mais 3 fatores de risco, provenientes do protocolo de Davison-Caprini: anestesia geral, malignidade, restrição ao leito por mais que 72 horas, com o objetivo de corrigir a carência de indicação medicamentosa nas cirurgias reparadoras;

- Administração da HBPM, quando indicada, apenas $12 \mathrm{~h}$ após a cirurgia, conforme o protocolo Davison-Caprini, buscando reduzir ao máximo o risco de sangramento.

\section{CONCLUSÃo}

Após a comparação entre os protocolos brasileiro e americano para profilaxia da TVP, observou-se que o brasileiro indica a profilaxia medicamentosa em uma parcela significativamente maior de pacientes, quando comparado ao americano, valorizando mais os procedimentos estéticos que os reparadores. Por outro lado, o americano indica a profilaxia química em uma parcela menor de pacientes, destacando, entretanto, mais as cirurgias reparadoras.

O protocolo proposto, Sandri M, mostrou-se equivalente ao protocolo Davison-Caprini na indicação geral da profilaxia medicamentosa e em sua indicação nas cirurgias reparadoras, mantendo-se equivalente ao protocolo Sandri no que se refere à indicação da profilaxia medicamentosa nas cirurgias estéticas, sendo, desta forma, mais abrangente.

Entretanto, estudos controlados e randomizados a respeito da prevalência de complicações tromboembólicas em cirurgias plásticas estéticas e reparadoras serão necessários para se verificar a validade desta nova proposta na prevenção de tais complicações.

\section{REFERÊNCIAS}

1. Reinisch JF, Bresnick SD, Walker JW, Rosso RF. Deep venous thrombosis and pulmonary embolus after face lift: a study of incidence and prophylaxis. Plast Reconstr Surg. 2001;107(6):1570-7.

2. Albin R, de Campo T. Large-volume liposuction in 181 patients. Aesthetic Plast Surg. 1999;23(1):5-15.

3. Grazer FM, Goldwyn RM. Abdominoplasty assessed by survey, with emphasis on complications. Plast Reconstr Surg. 1977;59(4):513-7.

4. van Uchelen JH, Werker PM, Kon M. Complications of abdominoplasty in 86 patients. Plast Reconstr Surg. 2001;107(7):1869-73.

5. Hatef DA, Trussler AP, Kenkel JM. Procedural risk for venous thromboembolism in abdominal contouring surgery: a systematic review of the literature. Plast Reconstr Surg. 2010;125(1):352-62.

6. Erdmann D, Sundin BM, Moquin KJ, Young H, Georgiade GS. Delay in unipedicled TRAM flap reconstruction of the breast: a review of 76 consecutive cases. Plast Reconstr Surg. 2002;110(3):762-7.
7. Hester TR Jr, Baird W, Bostwick J $3^{\text {rd }}$, Nahai F, Cukic J. Abdominoplasty combined with other major surgical procedures: safe or sorry? Plast Reconstr Surg. 1989;83(6):997-1004.

8. Voss SC, Sharp HC, Scott JR. Abdominoplasty combined with gynecologic surgical procedures. Obstet Gynecol.1986;67(2):181-5.

9. Olsson EH, Tukiainen E. Three-year evaluation of late breast reconstruction with a free transverse rectus abdominis musculocutaneous flap in a county hospital in Sweden: a retrospective study. Scand J Plast Reconstr Surg Hand Surg. 2005;39(1):33-8.

10. Spear SL, Ducic I, Low M, Cuoco F. The effect of radiation on pedicled TRAM flap breast reconstruction: outcomes and implications. Plast Reconstr Surg. 2005;115(1):84-95.

11. Broughton $\mathrm{G} 2^{\text {nd }}$, Rios JL, Rohrich RJ, Brown SA. Deep venous thrombosis prophylaxis practice and treatment strategies among plastic surgeons: survey results. Plast Reconstr Surg. 2007;119(1):157-74.

12. Caprini JA, Arcelus JI, Reyna JJ. Effective risk stratification of surgical and nonsurgical patients for venous thromboembolic disease. Semin Hematol. 2001;38(2 Suppl 5):12-9.

13. Davison SP, Venturi ML, Attinger CE, Baker SB, Spear SL. Prevention of venous thromboembolism in the plastic surgery patient. Plast Reconstr Surg. 2004;114(3):43E-51E.

14. Guyatt G, Schunemann HJ, Cook D, Jaeschke R, Pauker S. Applying the grades of recommendation for antithrombotic and thrombolytic therapy; the Seventh ACCP Conference on Antithrombotic and Thrombolytic Therapy. Chest. 2004;126(3 Suppl):179S-187S.

15. Hatef DA, Kenkel JM, Nguyen MQ, Farkas JP, Abtahi F, Rohrich RJ, et al. Thromboembolic risk assessment and the efficacy of enoxaparin prophylaxis in excisional body contouring surgery. Plast Reconstr Surg. 2008;122(1):269-79.

16. Sandri JL. Profilaxia do tromboembolismo em cirurgia plástica. In: Carreirão S, Cardim V, Goldenberg D, eds. Cirurgia Plástica - Sociedade Brasileira de Cirurgia Plástica. São Paulo: Atheneu;2005. p.119-25.

17. Nurmohamed MT, Rosendaal FR, Buller HR, Dekker E, Hommes DW, Vandenbroucke JP, et al. Low-molecular-weight heparin versus standard heparin in general and orthopaedic surgery: a meta-analysis. Lancet. 1992;340(8812):152-6.

18. Koch A, Bouges S, Ziegler S, Dinkel H, Daures JP, Victor N. Low molecular weight heparin and unfractionated heparin in thrombosis prophylaxis after major surgical intervention: update of previous meta-analyses. Br J Surg. 1997;84(6):750-9.

19. Newall G, Ruiz-Razura A, Mentz HA, Patronella CK, Ibarra FR, Zarak A. A retrospective study on the use of a low-molecular-weight heparin for thromboembolism prophylaxis in large-volume liposuction and body contouring procedures. Aesthetic Plast Surg. 2006;30(1):86-95.

20. Kim EK, Eom JS, Ahn SH, Son BH, Lee TJ. The efficacy of prophylactic low-molecular-weight heparin to prevent pulmonary thromboembolism in immediate breast reconstruction using the TRAM flap. Plast Reconstr Surg. 2009;123(1):9-12.

21. Geerts WH, Pineo GF, Heit JA, Bergqvist D, Lassen MR, Colwell CW, et al. Prevention of venous thromboembolism: the Seventh ACCP Conference on Antithrombotic and Thrombolytic Therapy. Chest. 2004;126(3 Suppl):338S-400S

22. Warkentin TE, Levine MN, Hirsh J, Horsewood P, Roberts RS, Gent $\mathrm{M}$, et al. Heparin-induced thrombocytopenia in patients treated with low-molecular-weight heparin or unfractionated heparin. N Engl J Med.1995;332(20):1330-5.

23. Hull RD, Brant RF, Pineo GF, Stein PD, Raskob GE, Valentine KA Preoperative vs postoperative initiation of low-molecular-weight heparin prophylaxis against venous thromboembolism in patients undergoing elective hip replacement. Arch Intern Med. 1999;159(2):137-41. 